\title{
Inactivated vaccines. 1. Volunteer studies with very high doses of influenza vaccine purified by zonal ultracentrifugation*
}

\author{
STEVEN R. Mostow $\dagger$ \\ M.D. \\ StePhen C. Schoenbaum $\ddagger$
M.D.
}

\author{
WALTER R. DOWDle \\ Ph.D.
}

MARION T. COLEMAN

Ph.D.

\author{
HAROLD S. KaYe \\ M.S. \\ Center for Disease Control, Health Services and Mental Health Administration, \\ Public Health Service, U.S. Department of Health, Education and Welfare, \\ Atlanta, Georgia 30333
}

\begin{abstract}
Summary
In these studies very high doses (up to $4800 \mathrm{CCA}$ units in adults) of formalin inactivated influenza vaccine purified by density gradient centrifugation were given safely to more than 5000 volunteers drawn from adolescent, middle aged and elderly populations. The relative paucity of reactions, compared with those groups receiving much lower doses of formalin-inactivated vaccine produced by Sharples centrifugation, suggests that reactions are due to non-viral substances rather than to toxic properties of the viruses, and that these substances are removable.

Homologous serum HI antibody responses increased with increasing vaccine dosage and there was no plateau effect at the higher dose level. In those groups studied, the appearance of neutralizing activity in post-vaccination nasal washings correlated closely with the higher vaccine doses and higher $\mathrm{HI}$ antibody titres.

The occurrence of the 'Hong Kong flu' epidemic in two of the groups, including over 3000 vaccine recipients, provided a unique opportunity to study the efficacy of the various vaccines used. Only those groups receiving high doses of a Hong Kong-like antigen (A2/Aichi/2/68) enjoyed significant reduction
\end{abstract}

\footnotetext{
* Presented by the senior author before the symposium on Influenza Vaccines, London, 27 April 1972.

$\dagger$ Present address: Cleveland Metropolitan General Hospital, Cleveland, Ohio 44109.

$\ddagger$ Present address: Peter Bent Brigham Hospital, Boston, Massachusetts 02115.

Requests for reprints: Walter R. Dowdle, Center for Disease Control, Respiratory Virology Unit, Atlanta, Georgia 30333.
}

in illness $(70 \%)$. Furthermore, there was a $90 \%$ reduction in the incidence of fever in that group.

These results indicate that very large doses of purified influenza vaccine can be given safely. Significant prevention and modification of disease caused by influenza via the vaccine approach is dependent on the administration of large doses of the appropriate? antigen.

IN a previous study (Kaye, Dowdle \& McQueen, 1969) we demonstrated in mice that protection against a broad spectrum of heterotypic influenza $\mathbf{A}$ (Asian) strains could be achieved with increasing concentrations of aqueous inactivated influenza vaccine. These results suggested that more concentrated doses of vaccine might also broaden the antibody response and provide better protection in man. Although the dose-related frequency of local and systemic reactions precluded the use of high concentrations of conventional Sharples centrifuged vaccines, initial reports of low toxicity of vaccine prepared by zonal ultracentrifugation were encouraging (Reimer et al., 1967; Peck, 1968).

Here we review some of our findings with volunteers inoculated with high doses of vaccine purified by zonal ultracentrifugation. For convenience the review is presented in two parts: first, the 1967-68 studies of increasing dosage of vaccine and its effect in terms of adverse reaction and antibody response in volunteers and, second, the 1968-69 studies of the efficacy of high doses of vaccine in preventing natural disease in man. Both of these studies are described in detail elsewhere (Mostow et al., 1969; Schoenbaum et al., 1969; Mostow et al., 1970). 
The effect of increasing dosage of influenza vaccine in terms of reactions and antibody responses in volunteers

\section{Methodology}

In this study, purified A/Japan/305/57 (H2N2) influenza vaccines were given subcutaneously in graded doses of 300-4800 CCA units. Standard Sharples vaccines at 300 and $600 \mathrm{CCA}$ units were employed as well as a control vaccine containing all components of zonal vaccine except the virus. The vaccines, method of production and dosage in CCA units are listed in Table 1.

\begin{tabular}{cc}
$\begin{array}{c}\text { TABLE 1. Inactivated A/Japan/305/57(H2N2) } \\
\text { influenza vaccines* }\end{array}$ \\
\hline \multicolumn{2}{c}{ Dosage (CCA units) } \\
\hline Sharples processing $\quad$ Zonal processing \\
\hline 300 & 0 (placebo) \\
600 & 300 \\
& 600 \\
& 2400 \\
& 3461 \\
& $4800 \dagger$
\end{tabular}

* Trials performed at Atlanta Federal Penitentiary, Atlanta, Georgia, U.S.A.

$\uparrow$ Two injections of 2400 CCA units.

Vaccines were administered in $1 \mathrm{ml}$ amounts by jet injector gun to volunteers from the Atlanta Federal Penitentiary. Because the adverse effects of very large doses of purified vaccines were not known, each dose increment was given on alternate days so that recipients could be observed for $48 \mathrm{hr}$ before the group receiving the next higher dose was vaccinated.

Serum and nasal specimens were collected before and 3 weeks after vaccination. Serum specimens were examined by haemagglutination inhibition (HI) tests, and nasal washings were examined by conventional virus neutralization tests (Robinson \& Dowdle, 1969).

Reactions to the vaccines were evaluated by questioning each volunteer before injection and 8 , 24 and $48 \mathrm{hr}$ afterwards about the occurrence of headache, eye pain, malaise, generalized muscle pain, loss of appetite, nausea and chills. Oral temperatures were also recorded. Measurements of erythema and induration at the injection site and complaints of local tenderness were recorded at 8 , 24 and $48 \mathrm{hr}$.

\section{Adverse reactions}

We observed no clear relationship between dosage or type of vaccine and a temperature change of $1.5^{\circ} \mathrm{F}$ or greater, or four or more symptoms reported at any interview (Table 2). However, the occurrence of local reactions was directly related to the concentration of the purified vaccines. Thus, the frequency of erythema $(>50 \mathrm{~mm})$ in the 3461 CCA unit group was nearly twenty times that in the $300 \mathrm{CCA}$ unit recipients. The incidence of induration $(>20 \mathrm{~mm})$ was nearly eight times greater in the high-dose group than in the $300 \mathrm{CCA}$ unit group. However, the occurrence of induration in the high-dose group was the same as that in Sharples vaccine group. The peak occurrence of local reactions was observed $24 \mathrm{hr}$ after vaccination and by $48 \mathrm{hr}$ had regressed substantially.

\section{Serologic responses}

Comparable doses of zonal purified vaccine and Sharples vaccine produced essentially the same serum antibody responses against the vaccine antigen (A/Japan/305/57) and against a virus isolated in an outbreak following the vaccination programme (A/Georgia/19/67). The removal of most of the nonviral protein by density gradient zonal ultracentrifugation neither decreased nor enhanced the antigenicity of the vaccines. To simplify the presentation, only those results from zonal vaccines are discussed. The percentage of four-fold or greater serum $\mathrm{HI}$ responses was dose-related. Whereas $68 \%$ of the 300 CCA unit group showed $a \geqslant$ four-fold response, $88 \%$ of the high dose recipients showed a similar response. The effects of increasing dosage are reflected in the geometric mean postvaccination titres for each group. Homologous titres (A/Japan/305/57)

TABLE 2. Inactivated influenza vaccines, Atlanta Federal Penitentiary, 1967-68. Occurrence of adverse reactions in recipients of A/Japan/305/57 (H2N2) vaccines

\begin{tabular}{|c|c|c|c|c|c|c|c|c|c|}
\hline \multirow[b]{2}{*}{ Dosage (CCA units) } & \multicolumn{2}{|c|}{ Sharples } & \multicolumn{5}{|c|}{ Zonal } & \multirow[b]{2}{*}{4800} & \multirow[b]{2}{*}{0 (Placebo) } \\
\hline & 300 & 600 & 300 & 600 & 1200 & 2400 & 3461 & & \\
\hline \multicolumn{10}{|l|}{ Local reactions $(\%)$} \\
\hline Erythema $>50 \mathrm{~mm}$ & 20 & 18 & 2 & 17 & 21 & 21 & 39 & - & 2 \\
\hline Induration $>20 \mathrm{~mm}$ & 1 & 36 & 4 & 15 & 25 & 30 & 39 & - & 2 \\
\hline \multicolumn{10}{|l|}{ Systemic reactions $(\%)$} \\
\hline Temperature change & & & & & & & & & \\
\hline of $>1 \cdot 5^{\circ} \mathrm{F}$ & 15 & 23 & 33 & 23 & 14 & 41 & 21 & 33 & 19 \\
\hline Four or more & 10 & 22 & 11 & & & & & & \\
\hline systemic reactions & 10 & 22 & 11 & 22 & 12 & 10 & 10 & 15 & 10 \\
\hline
\end{tabular}


increased with dosage as did the titres to a wide variety of antigens isolated up to 10 years after the vaccine strain. No plateau effect was observed, and titres of 5120, 10,240 and even 20,480 were not uncommon. The effects of increasing dosages of vaccine are more dramatically illustrated if we look at fold increase in geometric mean $\mathrm{H} 1$ titre. The 300 CCA unit vaccine produced a seven-fold increase in homologous geometric mean $\mathrm{HI}$ titre, whereas the 4800 CCA unit vaccine produced a thirty-five-fold increase (Fig. 1).

The presence of neutralizing substances in nasal washings after subcutaneous immunization also increased with vaccine dose (Fig. 2). The 300 CCA unit vaccine resulted in nasal-wash neutralizing activity in $40 \%$ of the recipients, whereas the 4800 CCA unit vaccine resulted in neutralizing activity in $80 \%$.

These results indicated that high doses of inactivated influenza vaccines prepared by zonal ultracentrifugation could be given safely to adults with essentially no greater adverse reaction rate than has been associated with standard doses of Sharples vaccines. Although the serum and nasal secretory antibody responses to the higher doses suggested that increased protection might be conferred upon the recipients, efficacy of the higher doses for prevention of natural infection remained to be proven.

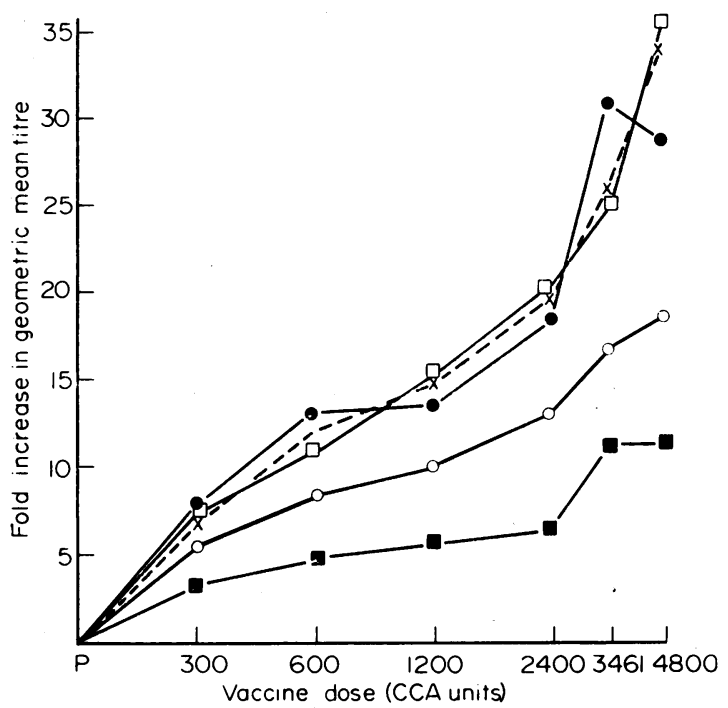

FIG. 1. Fold increase in geometric mean serum HI antibody titre with increasing dosages of $\mathrm{A} / \mathrm{Japan} / 305 / 57$ (H2N2) vaccines prepared by zonal ultracentrifugation. $\square-\square$, A/Japan/305/57 (H2N2); $\times \cdots \times$, A/Japan/ 170/62 (H2N2); -O, A/Taiwan/1/64 (H2N2); $\mathrm{O}-\mathrm{O}, \mathrm{A} / \mathrm{New}$ Jersey/1/67 (H2N2); $\square-\square, \mathrm{A} /$ Georgia/ 19/67 (H2N2).

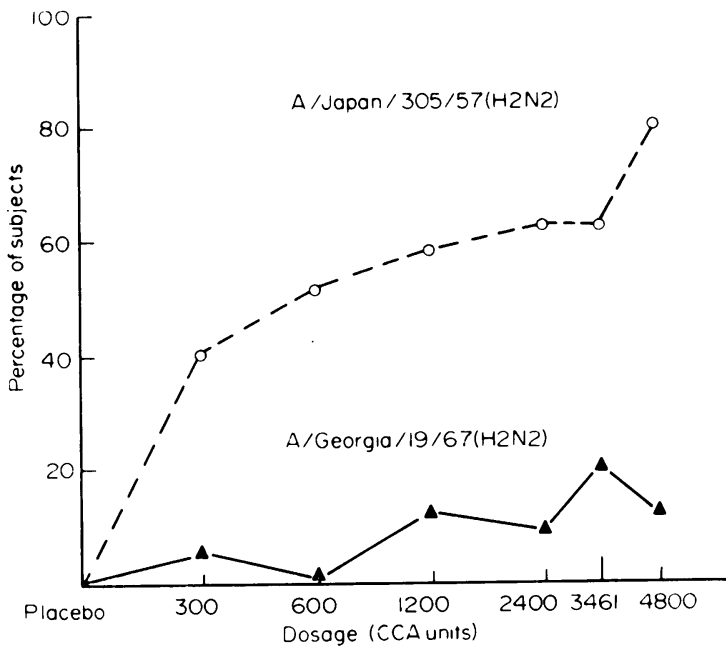

FIG. 2. Appearance of neutralizing substances in nasal secretions of volunteers receiving increasing dosages of A/Japan/305/57 (H2N2) vaccines prepared by zonal ultracentrifugation.

The efficacy of high doses of influenza vaccines, purified by zonal ultracentrifugation in preventing natural disease in man

Methodology

In this study purified A/Japan/170/62 (H2N2), A/Aichi/2/68 (H3N2), and B/Mass/3/66 vaccines at 300 and 3000 CCA units each were evaluated for adverse reactions and antibody responses in populations in three locations: the Georgia State Prison, secondary schools in California, and a retirement community in California. In addition, each of the vaccines was evaluated for effectiveness in prevention of illness caused by Hong Kong influenza in the Georgia State Prison and retirement community populations.

The methods of vaccine administration, collection and testing of specimens were similar to those described for previous trials (1967-68).

Reactions to the vaccines were also evaluated at the Georgia State Prison as described for the previous study. At the retirement community and schools, each vaccinee was given a questionnaire concerning the systemic and local reactions listed for the prisoners and was requested to complete and return it 4 days after vaccination. In addition, school absenteeism was tabulated for vaccinated and unvaccinated students for the 3 days immediately following injections.

\section{Adverse reactions}

In the Georgia State Prison local reactions to high doses of all the zonal purified vaccines were greater than those to the lower doses but, in general, 
were less severe than those associated with the Sharples vaccines (Table 3). Systemic reactions, including fever, were also relatively infrequent except for those receiving the $3000 \mathrm{CCA}$ unit $\mathrm{B} / \mathrm{Mass} / 3 / 66$ vaccine.

In the retirement community, two-thirds of those persons receiving a low dose of zonally purified vaccine reported no symptoms (Table 4), and nearly half of the high dose recipients had no reaction. In contrast, more than two-thirds of the recipients of the standard dose of Sharples vaccine had either a local or systemic reaction, or both.

Students recorded adverse reactions to the A vaccines twice as frequently as the adults at the retirement community (Table 5). However, there was no excess in school absenteeism in those groups receiving either the $\mathrm{A} / \mathrm{Aichi}$ or $\mathrm{A} / \mathrm{Japan}$ vaccines. This attests to the mildness of the reactions. In sharp contrast were reactions found among the recipients of the high dose $\mathrm{B} /$ Mass vaccine $(75 \%)$, thus con- firming the observations in the Georgia State Prison. In the first two schools, a four-fold increase in absenteeism occurred among the recipients of one of the vaccines on the day following vaccination. On the basis of this observation, we discontinued the administration of that vaccine in the remaining two schools. Absentee rates returned to normal on the second day after vaccination. Upon later decoding, this vaccine was identified as the high dose of B/Mass/3/66.

\section{Serologic responses}

The results from the Georgia State Prison, retirement community and Orange County schools are summarized in Table 6. Only the Aichi (Hong Kong variant) vaccine produced significant increases in antibody to the Aichi antigen. In fact, $76-88 \%$ of those receiving 300 CCA units of this vaccine had a four-fold or greater rise in serum $\mathrm{HI}$ titre. At the higher dose level (3000 CCA units), $88-96 \%$ of

TABLE 3. Adverse reactions to inactivated influenza vaccines, Georgia State Prison, 1968-69

\begin{tabular}{|c|c|c|c|c|c|c|c|}
\hline & \multicolumn{7}{|c|}{ Vaccine received (CCA units/ml) } \\
\hline & \multicolumn{2}{|c|}{ B/Mass/3/66 } & \multicolumn{2}{|c|}{ A/Japan/170/62 } & \multicolumn{2}{|c|}{ A/Aichi/2/68 } & \multirow{2}{*}{$\frac{\text { Polyvalent }}{600}$} \\
\hline & 300 & 3000 & 300 & 3000 & 300 & 3000 & \\
\hline \multicolumn{8}{|l|}{ Local reactions $(\%)$} \\
\hline Erythema $>50 \mathrm{~mm}$ & 4 & 20 & 2 & 16 & 0 & 9 & 33 \\
\hline Induration $>20 \mathrm{~mm}$ & 14 & 36 & 10 & 22 & 19 & 30 & 56 \\
\hline \multicolumn{8}{|l|}{ Systemic reactions $(\%)$} \\
\hline Fever $\geqslant 100^{\circ} \mathrm{F}\left(37.8^{\circ} \mathrm{C}\right)$ & 0 & 9 & 0 & 2 & 2 & 2 & 4 \\
\hline Fever $\geqslant 99.6^{\circ} \mathrm{F}\left(37.6^{\circ} \mathrm{C}\right)$ & 2 & 22 & 0 & 6 & 4 & 4 & 7 \\
\hline Four or more systemic reactions & 9 & 36 & 10 & 14 & 13 & 21 & 20 \\
\hline
\end{tabular}

TABLE 4. Adverse reactions to inactivated influenza vaccines, retirement community, 1968-69

\begin{tabular}{|c|c|c|c|c|c|c|}
\hline & \multicolumn{6}{|c|}{ Vaccine received (CCA units/ml) } \\
\hline & \multicolumn{2}{|c|}{ A/Aichi/2/68 } & \multicolumn{2}{|c|}{ A/Japan/170/62 } & \multirow{2}{*}{$\frac{\text { Divalent* }}{600}$} & \multirow{2}{*}{$\frac{\text { Polyvalent } \dagger}{600}$} \\
\hline & 300 & 3000 & 300 & 3000 & & \\
\hline No reactions $(\%)$ & 69 & 49 & 66 & 46 & 32 & 32 \\
\hline Any local reaction $(\%)$ & 24 & 44 & 22 & 44 & 63 & 62 \\
\hline Any systemic reaction $(\%)$ & 17 & 28 & 17 & 28 & 33 & 39 \\
\hline Local and systemic reactions $(\%)$ & 9 & 21 & 5 & 18 & 28 & 33 \\
\hline
\end{tabular}

* Containing A/Japan/170/62 (H2N2), A/Taiwan/1/64 (H2N2) and B/Mass/3/66.

$\dagger$ Containing A/Japan/170/62, A/Taiwan/1/64, A/Ann Arbor/1/57 (H1N1) and B/Mass/3/66.

TABLE 5. Adverse reactions to inactivated influenza vaccines, Orange County Schools, 1968-69

\begin{tabular}{|c|c|c|c|c|c|c|}
\hline & \multicolumn{6}{|c|}{ Vaccine received (CCA units/ml) } \\
\hline & \multicolumn{2}{|c|}{ A/Aichi/2/68 } & \multicolumn{2}{|c|}{ A/Japan/170/62 } & \multicolumn{2}{|c|}{ B/Mass/3/66 } \\
\hline & 300 & 3000 & 300 & 3000 & 300 & 3000 \\
\hline No reactions $(\%)$ & 34 & 23 & 35 & 23 & 36 & 17 \\
\hline Any local reaction $(\%)$ & 49 & 61 & 51 & 70 & 50 & 72 \\
\hline Any systemic reaction $(\%)$ & 37 & 56 & 41 & 47 & 44 & 75 \\
\hline Local and systemic reactions $(\%)$ & 23 & 39 & 30 & 40 & 30 & 64 \\
\hline
\end{tabular}


adults had a four-fold or greater response. Also, the Aichi vaccines were as effective as the homologous A/Japan vaccines in stimulating A/Japan antibody.

Recipients of A/Japan and B/Mass vaccines had fewer homologous four-fold rises than the Aichi vaccines. This probably reflects previous exposure to strains similar to A/Japan and B/Mass, either through natural infection or vaccination. Prevaccination titres to the A/Japan antigen were high (Table 6b), whereas prevaccination titres to the Aichi antigen were low $(<10 \mathrm{GMT})$, reflecting the lack of exposure to this virus in the past. As in the Atlanta Federal Penitentiary study the year before, the higher doses of $A$ vaccines stimulated large increases in GMT.

\section{Vaccine efficacy}

In late December 1968, the physician-in-charge reported a sharp increase in febrile respiratory disease at the Georgia State Prison. The Hong Kong variant was isolated from the throat swabs of thirty of forty-two prisoners seen at sick call. All forty-two had diagnostic serologic rises to the Hong Kong antigen. In addition, $74 \%$ of those who said they had an influenza-like illness on interview demonstrated four-fold or greater rises in antibody to the Hong Kong virus. Details of surveillance procedures and methods of assessing efficacy have been previously reported (Schoenbaum et al., 1969).

Comparison of the attack rates in the groups which received different vaccines revealed the following (Table 7):

(1) The attack rate among inmates receiving 300 CCA units of B/Mass vaccine were similar to those among men receiving the $300 \mathrm{CCA}$ unit A/Japan vaccine. These similarities also were seen among the 3000 CCA unit recipients of the same vaccines.

(2) Based upon the criteria of history of a febrile illness, going to bed, or attendance at sick call, the attack rates for the $3000 \mathrm{CCA}$ unit B/Mass or $\mathrm{A} / \mathrm{Japan}$ vaccine were lower than for recipients of the 300 CCA unit dose of these vaccines, but these differences were not statistically significant $(P>0.05)$.

TABLE 6. Haemagglutination inhibition antibody responses in study populations receiving subcutaneous doses of commercial influenza (Sharples) vaccines and high and low doses of purified (zonal) influenza vaccines

\begin{tabular}{|c|c|c|c|c|c|c|c|c|c|}
\hline \multirow{3}{*}{ Vaccines } & \multicolumn{9}{|c|}{ Test antigens } \\
\hline & \multicolumn{3}{|c|}{ A/Aichi/2/68 (H3N2) } & \multicolumn{3}{|c|}{ A/Japan/170/62 (H2N2) } & \multicolumn{3}{|c|}{$\mathrm{B} /$ Mass $/ 3 / 66$} \\
\hline & GSP* & CRC & OCS & GSP & CRC & OCS & GSP & CRC & OCS $\stackrel{\bar{c}}{\underline{7}}$ \\
\hline
\end{tabular}

(a) Percentage of recipients with $\geqslant$ four-fold $\mathrm{HI}$ antibody rises

Zonal:

$$
\begin{array}{r}
\text { A/Aichi/2/68 } \\
300 \text { CCA } \\
3000 \text { CCA } \\
\text { A/Jap } / 170 / 62 \\
300 \text { CCA } \\
3000 \text { CCA } \\
\text { B/Mass/3/66 } \\
300 \text { CCA } \\
3000 \text { CCA }
\end{array}
$$

$\begin{array}{rrr}78 & 76 & 88 \\ 93 & 88 & 96 \\ & & \\ 15 & 0 & \\ 16 & 3 & 23 \\ & & \\ 0 & & \end{array}$

Sharples:

Divalent

(600 CCA unit recipients only)

Polyvalent

(600 CCA unit recipients only)

$11 \quad 12$

$\begin{array}{rr}88 & 6 \\ 96 & 67 \\ 6 & 40 \\ 23 & 5 \\ 3 & \\ 3 & \end{array}$

62
67
40
57

3
5

$\begin{array}{ll}20 & 7 \\ 45 & 9 \\ 43 & 67 \\ 53 & 8\end{array}$

$\begin{array}{ll}71 & 7 \\ 92 & 5 \\ 67 & 5 \\ 80 & 5 \\ 2 & 23 \\ 8 & 60\end{array}$

$\begin{array}{rrr}7 & 0 & 1 \\ 5 & 3 & 4 \\ & & \\ 5 & 3 & 12 \\ 5 & 3 & 12 \\ & & \\ 23 & & 66 \\ 60 & & 71\end{array}$

(b) Geometric mean $\mathrm{HI}$ titres before and after immunization

Zonal:

$$
\begin{array}{r}
\text { A/Aichi/2/68 } \\
300 \text { CCA } \\
3000 \text { CCA } \\
\text { A/Jap } / 170 / 62 \\
300 \text { CCA } \\
3000 \text { CCA } \\
\text { B/Mass } / 3 / 66 \\
300 \text { CCA } \\
3000 \text { CCA }
\end{array}
$$

Sharples:

Divalent

Polyvalent

$$
\begin{aligned}
& </ 69^{*}</ 60</ 64 \\
& </ 126</ 229</ 153 \\
& <1<\quad<1<\quad<1< \\
& <1<\quad<1<\quad<1< \\
& \begin{array}{l}
<1< \\
<1<
\end{array} \\
& \begin{array}{l}
<1< \\
<1<
\end{array}
\end{aligned}
$$

$81 / 456$

$99 / 728$

$29 / 54$

$32 / 103$

$37 / 282$

$37 / 640$

$80 / 202 \quad 26 / 71 \quad 36 / 234$

$101 / 490$

$34 / 108$

$41 / 432$

$92 / 86$

$90 / 111$

$<1<\quad<1<$

$\begin{array}{ll} & 22 / 58 \\ 86 / 202 & 32 / 75\end{array}$

$38 / 53$
$12 / 15$

$16 / 19$

$10 / 10$

$12 / 14$

$15 / 29$

$12 / 53$

48

39

* GSP, Georgia State Prison; CRC, California retirement community; OCS, Orange County schools.

$\dagger<$, Negative test at a $1: 10$ dilution of serum. 
TABLE 7. Efficacy of inactivated influenza vaccines. Attack rates computed from questionnaires and sick-call surveillance, Georgia State Prison, 1968-69

\begin{tabular}{|c|c|c|c|c|c|c|c|}
\hline & \multicolumn{7}{|c|}{ Attack rates $(\%)$} \\
\hline & \multicolumn{2}{|c|}{$\begin{array}{l}\text { A/Aichi/2/68 } \\
\text { (CCA units) }\end{array}$} & \multicolumn{2}{|c|}{$\begin{array}{c}\text { A/Japan } / 170 / 62 \\
\text { (CCA units) }\end{array}$} & \multicolumn{2}{|c|}{$\begin{array}{l}\text { B/Mass/3/66 } \\
\text { (CCA units) }\end{array}$} & \multirow{2}{*}{$\begin{array}{c}\begin{array}{c}\text { Polyvalent* } \\
\text { (CCA units) }\end{array} \\
600\end{array}$} \\
\hline & 300 & 3000 & 300 & 3000 & 300 & 3000 & \\
\hline \multicolumn{8}{|l|}{ Questionnaire: } \\
\hline Illness & 32 & 15 & 41 & 41 & 39 & 40 & 68 \\
\hline Fever & 26 & 10 & 41 & 38 & 39 & 29 & 59 \\
\hline Confinement to bed & 12 & 3 & 31 & 16 & 32 & 17 & 51 \\
\hline \multicolumn{8}{|l|}{ Prison records: } \\
\hline Attendance at sick call & 17 & 10 & 42 & 19 & 43 & 24 & 33 \\
\hline \multicolumn{8}{|l|}{ Serology $(\mathbf{S} 2-\mathbf{S} 3) \dagger$ : } \\
\hline Four-fold rise to & & & & & & & \\
\hline A/Georgia/25/69‡ & 15 & 10 & 62 & 43 & 70 & 47 & 61 \\
\hline Four-fold rise to both & & & & & & & \\
\hline $\begin{array}{l}\text { A/Georgia/25/69 and } \\
\text { A/Aichi/2/68 }\end{array}$ & 9 & 5 & 44 & 30 & 59 & 44 & 50 \\
\hline No. of persons in group & 35 & 39 & 41 & 37 & 30 & 38 & 39 \\
\hline
\end{tabular}

* Containing A/Japan/170/62, A/Taiwan/1/64, A/Ann Arbor/1/57 (H1N1) and B/Mass/3/66.

$+\mathrm{S} 2$, post vaccine, pre-epidemic serum; S3, post epidemic serum.

$\ddagger$ Virus isolated during prison epidemic.

TABLE 8. Efficacy of inactivated influenza vaccines. Attack rates computed from questionnaires and medical clinic surveillance, California retirement community, 1968-69

\begin{tabular}{|c|c|c|c|c|c|c|c|}
\hline & \multicolumn{7}{|c|}{ Attack rates $(\%)$} \\
\hline & \multicolumn{2}{|c|}{$\begin{array}{l}\text { A/Aichi/2/68 } \\
\text { (CCA units) }\end{array}$} & \multicolumn{2}{|c|}{$\begin{array}{l}\text { A/Japan } / 170 / 62 \\
\text { (CCA units) }\end{array}$} & \multirow{2}{*}{$\frac{\begin{array}{c}\text { Divalent* } \\
\text { (CCA units) }\end{array}}{600}$} & \multirow{2}{*}{$\frac{\begin{array}{c}\text { Polyvalent } \dagger \\
\text { (CCA units) }\end{array}}{600}$} & \multirow[t]{2}{*}{$\begin{array}{l}\text { No vaccine } \\
\text { received }\end{array}$} \\
\hline & 300 & 3000 & 300 & 3000 & & & \\
\hline \multicolumn{8}{|l|}{ Questionnaire: } \\
\hline illness & 10 & 6 & 13 & 12 & 12 & 14 & 10 \\
\hline Febrile illness & 6 & 4 & 9 & 8 & 8 & 11 & 8 \\
\hline $\begin{array}{l}\text { Confinement to } \\
\text { bed }\end{array}$ & 7 & 5 & 12 & 10 & 9 & 12 & 8 \\
\hline \multicolumn{8}{|l|}{$\begin{array}{l}\text { Surveillance: } \\
\text { Attendance at }\end{array}$} \\
\hline $\begin{array}{l}\text { Fever at time of } \\
\text { clinic visit }\end{array}$ & $1 \cdot 2$ & 0.7 & $2 \cdot 2$ & $1 \cdot 8$ & $2 \cdot 2$ & $3 \cdot 5$ & $1 \cdot 7$ \\
\hline $\begin{array}{l}\text { No. of persons in } \\
\text { group }\end{array}$ & 576 & 596 & 577 & 611 & 600 & 566 & 848 \\
\hline
\end{tabular}

* Containing A/Japan/170/62 (H2N2), A/Taiwan/1/64 (H2N2) and B/Mass/3/66.

$\dagger$ Containing A/Japan/170/62, A/Taiwan/1/64, A/Ann Arbor/1/57 (H1N1) and B/Mass/3/66.

(3) The recipients of the $300 \mathrm{CCA}$ unit A/Aichi vaccine showed a lower attack rate than recipients of the B/Mass or A/Japan vaccines, but again these differences were not statistically significant $(P>0.05)$.

(4) Recipients of the $3000 \mathrm{CCA}$ unit A/Aichi vaccine showed a $63 \%$ reduction in attack rate when compared to the $\mathrm{B} / \mathrm{Mass}$ vaccinees.

(5) The 3000 CCA unit A/Aichi recipients, if ill had greatly modified disease as manifested by the very few men who had to go to bed because of illness.
(6) The attack rates among the recipients of standard, commercial polyvalent vaccine were higher than rates among recipients of any other vaccine, or among inmates not participating in the vaccine programme.

The epidemic at the retirement community, an open population, should better reflect the performance of the vaccine in a natural epidemic. Using the attack rates among the recipients of the 300 CCA units of A/Japan vaccine as a baseline, we observed the following (Table 8): 
(1) The attack rates of 300 and 3000 CCA unit A/Japan/170/62 vaccine recipients were essentially the same.

(2) The recipients of the commercial polyvalent vaccine again showed the highest attack rates.

(3) The reduction of illness by the 300 CCA unit A/Aichi vaccine was not statistically significant $(P>0.05)$.

(4) The nearly $60 \%$ reduction in attack rate by the 3000 CCA unit A/Aichi vaccine was significant $(P<0.05)$.

(5) The 3000 CCA unit A/Aichi vaccine recipients who were ill experienced less severe illness than those in the other vaccine groups.

\section{Discussion}

In this series of human volunteer trials, we have demonstrated that unusually high doses of influenza vaccine purified by zonal centrifugation can be given safely to both adults and older children. Although the students in the 1968 trials reported reactions twice as frequently as adults, class attendance was not affected except among those receiving the high dose of $B / M a s s / 3 / 66$ vaccine. The reactions associated with this vaccine may have been in some way related to the manufacture of this single vaccine lot. However, the possibility that $\mathrm{B} /$ Mass vaccine might be inherently more toxic than $\mathrm{A}$ vaccines cannot be ruled out. New criteria for safety and purity may be possible now that vaccines of increased concentration and purity are available. The removal of nonviral material through zonal centrifugation did not detract from the antibody response, suggesting that a local reaction is not necessary for an adjuvant effect. Stepwise increases in vaccine concentration produced stepwise increases in serum antibody. The higher concentrations of vaccine in the 1967-68 study also stimulated the appearance of neutralizing substances in the nasal secretions. In the 1968-69 trials, we were able to demonstrate a protective effect of the high (3000 CCA unit) Aichi vaccine against illness caused by the Hong Kong virus. Calculations of vaccine efficacy were remarkably similar for the two populations separated by 2000 miles. Using both the $\mathbf{B} /$ Mass and $\mathbf{A} / \mathbf{J} a p a n$ groups as a baseline, we determined that the protective efficacy of the 3000 CCA unit Aichi vaccine was $60-70 \%$. No significant protection was afforded by the standard vaccine dose. Of those persons in both populations who received the high dose of Aichi vaccine, relatively fewer had to be confined to bed or consult a physician. Among those who had fever, relatively fewer had high fevers. Thus, the high-dose vaccine was associated with less severe illness and with a reduction in confinement to bed.

We have demonstrated that increased dosages of highly purified vaccines can be given safely to adults and older children and that these dosages provide substantially better protection against illness than the previously recommended standard dose.

\section{References}

Kaye, H.S., Dowdle, W.R. \& McQueen, J.L. (1969) Studies on inactivated influenza vaccines. 1. The effect of dosage on antibody response and protection against homotypic and heterotypic influenza virus challenge in mice. American Journal of Epidemiology, 90, 162.

Mostow, S.R., Schoenbaum, S.C., Dowdle, W.R., ColeMAN, M.T. \& KAYE, H.S. (1969) Studies with inactivated influenza vaccines purified by zonal ultracentrifugation. 1. Adverse reactions and serological responses. Bulletin of the World Health Organization, 41, 525.

Mostow, S.R., Schoenbaum, S.C., Dowdle, W.R., Coleman, M.T., Kaye, H.S. \& Hierholzer, J.C. (1970) Studies on inactivated influenza vaccines. 2 . Effect of increasing dosage on antibody responses and adverse reactions in man. American Journal of Epidemiology, 92, 248.

PeCK, F.B. (1968) Purified influenza virus vaccine. Journal of the American Medical Association, 206, 2277.

Reimer, C.B., Baker, R.S., Van Frank, R.M., Newlin, T.E., Cline, G.B. \& Anderson, N.G. (1967) Purification of large quantities of influenza virus by density gradient centrifugation. Journal of Virology, 1, 1207.

Robinson, R.Q. \& Dowdle, W.R. (1969) Diagnostic Procedures for Viral and Rickettsial Diseases, 4th edn, p. 414. American Public Health Association, New York.

Schoenbaum, S.C., Mostow, S.R., Dowdle, W.R., ColeMAN, M.T. \& KAYE, H.S. (1969) Studies with inactivated influenza vaccines purified by zontal centrifugation. 2. Efficacy. Bulletin of the World Health Organization, 41, 531 . 